\title{
Clinical effects of a topical twin-serum (aqua phase and lipo phase) on skin radiance and wrinkle depth: role of active ingredients and delivery optimisation
}

\begin{abstract}
Introduction: Skin appearance plays as an important moderator in human daily social interactions so everyday we see new and innovative solutions to fight the signs of aging. One of the important parameters of skin appearance is skin complexion radiance as it could reflect the general health, emotional state, hormonal status, nutrition, fatigue, age or environmental factors such as smoking, drinking, seasons, etc.
\end{abstract}

Objective: The objective of the study was to evaluate the effect of the daily application of a bi-phasic serum during 8 weeks on the skin radiance and skin aging parameters of healthy women with dull complexion.

Subjects and method: 30 healthy female volunteers of 35 to 60 years old were enrolled in the study. The activated product (aquaserum and liposerum) were applied to the face, twice a day for 8 weeks. The evaluation was performed on T0 and T8 weeks by measuring the skin radiance using clinical scoring C.L.B.T. ${ }^{\mathrm{TM}}$ and Glossymeter ${ }^{\circledR}$ GL200, Crow's feet wrinkles by Bazin visual perioral scale, skin elasticity by Cutometer ${ }^{\circledR}$ SM575, and also the subject's self-assessment.

Results: All 30 subjects finished the study without any serious adverse events. The results revealed a significant improvement in all C.L.B.T. ${ }^{\mathrm{TM}}$ indicators presenting skin radiance through an increase of Luminosity (29\%), Transparency (28\%), Brightness (27\%) $(\mathrm{p}=0.001)$ while a decrease of Pink Red (38\%), Olive $(31 \%)$, Beige $(30 \%)$, Light Pink $(21 \%)$ parameters $(\mathrm{p}<0.001)$.For skin relief, statistical analyses revealed a significant decrease of wrinkles' depth evaluated by clinical scoring $(9 \%, \mathrm{p}=0.003)$. For the elasticity, an improvement which tends to be significant $(0.462 \pm 0.098$ at $\mathrm{T} 0$ and $0.506 \pm 0.111$ at $\mathrm{T} 8$ weeks; $\mathrm{p}=0.06$ ) for the Ur/Ue parameter, was observed. After 8 weeks of topical application of Perfectil® Twin-Serum, the subjects significantly found that their skin was less dull $(p=0.002)$, less red $(p=0.002)$, with less spots $(p=0.044)$, less sagging $(p=0.002)$ and less wrinkles $(\mathrm{p}=0.002)$.

Conclusion: The results observed in this trial showed that 8 weeks of Perfectil ${ }^{\circledR}$ Twin Serum use provide beneficial effect on skin radiance and skin surface relief (wrinkle depth). The action of the bi-phasic product may have a positive effect on the immediate penetration of the product which should be evaluated through the further studies. Furthermore, the ingredients seemed to restore the skin lipidic layer, which explains its protective effect during winter time.

Keywords: bi-phasic serum, C.L.B.T. ${ }^{\mathrm{TM}}$, skin elasticity, skin radiance
Volume 5 Issue 3 - 2021

\author{
Adeline Jeudy, Ferial Fanian, Thomas \\ Lihoreau, Philippe Humbert \\ Research and Studies Center on the Integument (CERT), \\ Department of Dermatology, Clinical Investigation Center (CIC \\ INSERM 143I), Besançon University Hospital, France
}

\begin{abstract}
Correspondence: Ferial Fanian, Dermatologist, Clinical Investigation Center (CIC INSERM I43I), Besançon University Hospital, France, Email fanian@gmail.com
\end{abstract}

Received: May II, 202 I | Published: May 26, 202 I

\section{Introduction}

Skin appearance plays as an important moderator in human daily social interactions so every day we see new and innovative solutions to fight the signs of aging. One of the important parameters of skin appearance is skin complexion radiance as it could reflects the general health, emotional state, hormonal status, nutrition, fatigue, age or environmental factors such as smoking, drinking, seasons, etc.

In 2001, Fink et $\mathrm{al}^{1}$ show that the skin texture plays a significant role in the judgment of female facial beauty, while it is also an index of fertility and health. In addition, the attractiveness ratings of the images judged by the male participants related positively to the parameters of skin homogeneity.

Furthermore, other studies revealed that the skin pigmentation and also the skin surface topography indices, particularly in women, have a significant influence on attractiveness judgements, as they seem primarily to be the markers of the subject's age and health. Recently, it has been shown that facial skin colour distribution significantly influences the perception of age and attractiveness of female faces, independent of skin surface topography cues. These cues account for a large proportion in facial age perception, whereas skin colour distribution seems to be a stronger health cue. ${ }^{2,3}$

Even small changes in skin surface topography affect the perceptions of a woman's facial age and attractiveness. ${ }^{4,5}$ The most important attributes to estimate age are eyes, lips and skin color uniformity. The eye area and the skin color uniformity were the main attributes related to perceived age. ${ }^{6}$

Jones et al. showed that colour variation in facial skin is a cue for health perception in female faces. ${ }^{7}$ Fink et al. ${ }^{8}$ showed that the variation in visible skin colour distribution (independent of facial form and skin surface topography) is able to selectively attract people's attention toward female faces, and this higher attention results in more positive statements about a woman's face. 
This review of literature shows that the homogeneity of colour, complexion and texture of the skin are important parameters for attractiveness and therefore self-esteem. Therefore, the cosmetics industries interested in beauty and improving the attractiveness try to develop cosmetic products to improve the homogeneity, skin colour and skin surface, the components of the skin radiance.

Skin radiance is often defined as a psychophysical parameter, being the mirror of general health, both in terms of physical and psychological condition. ${ }^{9}{ }^{10}$ A grey skin may reflect bad looks because of great fatigue, weariness, imbalanced hormonal status, negative emotional states (sadness, stress), illness, nutrition deficiency, tobacco, alcohol, pollution and seasonal effects. ${ }^{11-15}$ Inversely "peachy-coloured skin" reflects a smooth, pink and velvety skin and good health. ${ }^{16}$

Skin radiance is referred to these parameters without any precise definition and quantifiable appreciation data. All the parameters that constitute it and their relative proportions are difficult to list; they involve complex surface and internal qualities of the skin. ${ }^{17}$ In 2006, Baret et al. ${ }^{18}$ organized a round table meeting with women. As the result of these exchanges, many items (including the skin grain, brightness and colour) were selected and studied in volunteers of different age groups from image analysis of video data of their face. Experts performed clinical scoring and then divided the volunteers in two groups: the young one, whose radiance was considered more dependent on skin luminosity and colour ("rosy colour") and the old one, who was mainly characterized by irregular skin surface aspects and colour variations. These definitions corroborate the idea that radiance is multifactorial and naturally includes an important subjective part. ${ }^{17}$

The skin radiance seems to be a balanced mixture of: ${ }^{16,17,19}$

colour, itself mostly influenced by the skin microcirculation and inner skin structures, light reflection and more globally texture of the skin surface.

\section{Objectives}

The main objective of the study was to evaluate the effect of the daily application of a twin serum containing both lipo and aqua phase, during 8 weeks on the skin radiance and skin aging parameters of healthy women with dull complexion.

\section{Material and methods}

\section{Study design}

This study was a single center, open label, self comparative study on 30 healthy female volunteers with signs of skin photoaging. The mixed active product (aquaserum and lipiserum) were applied to the face, twice a day during 8 weeks. The recruitment of 30 volunteers (healthy females) was conducted at the Department of Dermatology, University Hospital of Besançon, France (French Health Ministry authorization $09023 \mathrm{M}$ and $09023 \mathrm{~S}$ for the conduct of clinical studies with drugs, medical devices, and cosmetic products). This investigation was approved by the internal ethics committee of Department of Dermatology, University Hospital of Besançon, France respecting the ICH-GCP. All volunteers signed an informed consent after having carefully read the modalities and the aim of the study.

\section{Subject selection}

The volunteers aged 35 to 60 years old presenting a Fitzpatrick phototype I to IV and a moderate to severe facial skin photoaging (Glogau grade 3 or 4 ), without sun or UV exposure 48 hours before the first day or during whole study duration. They were considered as healthy after a medical exam and not participating in any other study during the trial period.

Exclusion criteria were pregnancy, lactation, change of hormonal treatment during the last 3 months and during the study period (including HRT (Hormone Replacement Treatment) and recently started or any modification of contraceptive pill), hypersensitivity to one or more ingredients of the study product, serious acute or chronic illness, skin disease, or systemic or topical treatment with the potential interference with the results of the study.

\section{Study product}

A topical natural oil base serum was investigated (Perfectil ${ }^{\circledR}$ Twin Serum, Vitabiotics Ltd - London), consisting of two phases which are combined by the user immediately prior to application to form a fresh liposome emulsion:

\section{Lipo phase (Lipo Serum)}

contains several oils and vitamins which restore the lipidic layer of stratum conrneum to re-enforce the skin barrier function to keep more water in deep layers of the skin. Some of the ingredients are Triticum Vulgare (Wheat) Germ Oil, Persea Gratissima (Avocado) Oil, Lecithin, Caprylic/Capric Triglyceride, Tocopherol, Helianthus Annuus (Sunflower) Seed Oil, Ascorbyl Tetraisopalmitate, Retinyl Palmitate, Rosa Damascena Flower Oil, Citrus Aurantium Dulcis (Orange) Peel Oil, Limonene, Coenzyme Q10, Citronellol, Jasminum Officinale (Jasmine) Flower Extract, Calendula Officinal is Flower Extract, Lavandula Angustifolia (Lavender) Oil, Geraniol, Acmella Oleracea Extract, Linalool, Carthamus Tinctorius (Safflower) Seed Oil (lutein), Citral, Eugenol, Xanthophyll, Ascorbyl Palmitate.

\section{Aqua Phase (Aqua Serum)}

includes Aqua (Water), Panthenol (provitamin B5), Niacinamide (vitamin B3 or PP), Citrus Limon (Lemon) Peel Oil, Limonene; said to restore damaged epithelium, reduce itching and inflammation, improve skin hydration, reduces transepidermal water transepidermal water loss, improves skin roughness.

\section{Treatment procedure}

The products were applied twice daily, on the face for 8 weeks. The volunteers were required to mix two serums (the Lipi Serum and Aqua Serum ) on the palm of their hands with 1:3 ratio respectively, with a gentle massage for 1 minute before applying to the face. The total amount of applied product was according to the subject's facial surface between 2:6 to 4:12 drops of each phase.

During the study, each volunteer was asked not to change their hygiene products, not to expose themselves to the sun or UV sessions, not to apply any other products (cream, peelings, self-tanner) or any medications on their face, except for their routine makeup that was allowed at least 10 minutes after the penetration of serum.

\section{Treatment evaluation}

\section{Measuring constraints}

In order to homogenize the subjects' skin before the baseline measures, subjects were recruited at D-7 in order to pass a 7 day wash out period. During this period, the subjects were required to use a basic cleansing gel for their daily skin care.

The visits were performed at the Center for Studies and Research on the Integument (CERT) at the Department of Dermatology of 
University Hospital of Besançon. Measurements were performed at a regulated temperature of $22^{\circ} \mathrm{C} \pm 1^{\circ} \mathrm{C}$ and a regulated humidity of $55 \% \pm 10 \%$. The volunteers rested quietly for at least 15 minutes in stable environmental conditions prior to the assessment. The skin was not subject to any strain or stress. The study zone for all measurements was the face. Measurements zones were recorded using a flexible transparent plastic mask. This allowed the measurements to be done precisely in the same place on the face at each visit (Thermocell-Test ${ }^{\circledR}$ professional kit, Monaderm, Monte Carlo, Monaco). All subjects were instructed to wash their face in the evening of the day before the examination days (T0 and T8 weeks) and not to apply anything to their face (including water, soap, shower gel, cream, or makeup) on the day of the measuring visit, and not to take any coffee, tea or alcoholic beverages 2 hours before visits.

\section{Skin radiance}

A clinical scoring of skin radiance was performed according to a visual analysis scale (C.L.B.T. ${ }^{\mathrm{TM}}$, Musnier et al. ${ }^{20}$ including seven descriptors that evaluate 4 different colours (pink red, olive, beige, light pink) between $10 \%$ and $100 \%$ and 3 different parameters (skin transparency, brightness and luminosity) on a $0-10$ scale (Figure 1).

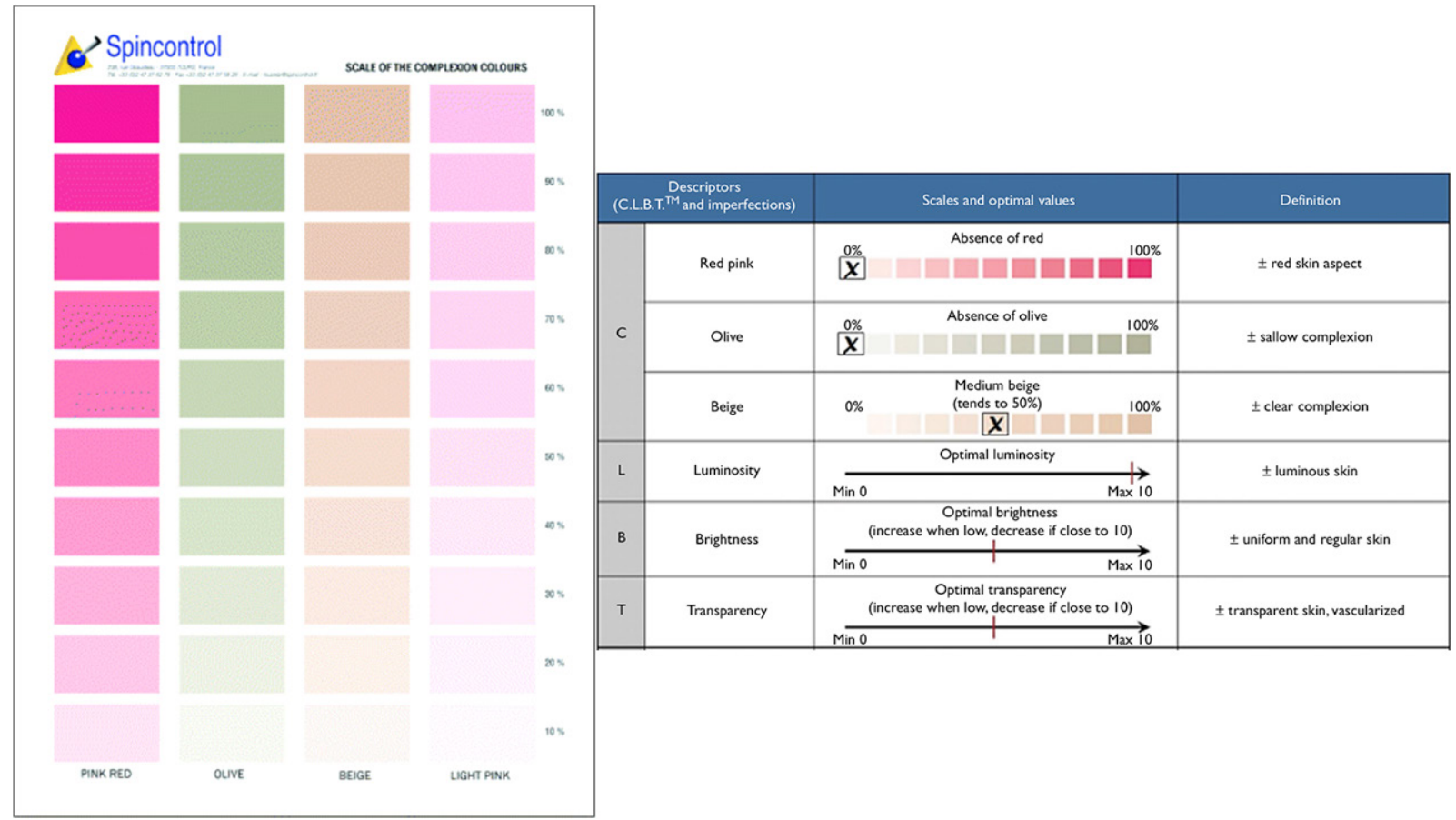

Figure I C.L.B.T descriptors.

The skin reflection was evaluated with the Glossymeter ${ }^{\circledR}$ GL200 (Courage and Khazaka, Köln, Germany) to assess the quantity of specular and diffuse light reflected by the skin. The probe head contains parallel white light created by LEDs. Light is sent out at $0^{\circ}$ and reflected by the mirrors to $60^{\circ}$ to the skin surface. A part of the light is directly reflected in the same angle, so the angle of incidence is equal to the angle of reflection, and the other part of the light is absorbed by the surface and then scattered and reflected diffusely. The Skin-Glossymeter ${ }^{\circledR}$ GL200 measures both the portion of directly reflected light, mirrored from the surface, which is related to the gloss and the scattered portion from the surface. Two separate measurement channels measure the direct reflected light (again guided by a mirror in the same angle, i.e. at $120^{\circ}$ ) and the diffuse reflected (scattered) light degrees. The scattered/diffuse reflected light is measured at $90^{\circ}$ (completely vertically above the measured surface) under the assumption that light is scattered in the same way over all degrees.

\section{Skin elasticity}

The skin elasticity was evaluated with the Cutometer ${ }^{\mathbb{B}}$ SM575 (Courage and Khazaka, Köln, Germany). The Cutometer ${ }^{\circledR}$ measures the elasticity of the skin in vivo according to the suction mechanism. A probe of $6 \mathrm{~mm}$ diameter is applied to the skin and a temporary vacuum is created (400mbars), thus deforming the skin (on time: 3 seconds; off time: 2 seconds; repetition: 5). Adhesive tape is attached to the probe and prevents the adjacent skin from being sucked into the chamber. The device holds a spring ensuring a constant application pressure. An optical system made of a light source and a receptor determines the skin deformation and its slackness. Different parameters are measured: immediate deformation $\mathrm{Ue}$ (U extensibility), delayed deformation $\mathrm{Uv}$ (U viscous), final deformation Uf ( $\mathrm{Uf}=\mathrm{Ue}+\mathrm{Uv}$ ) characterizes the firmness, and deformation of immediate retraction Ur, when the vacuum is removed. The ratio $\mathrm{Ur} / \mathrm{Ue}$ characterizes the elasticity of the skin and is independent of the thickness of the skin (Figure 2).

\section{Crow's feet wrinkles}

Crow's feet wrinkles were assessed by the standard visual clinical scoring: Bazin periorbital wrinkle scale. A scale of 1 to 4 points was considered for wrinkles' number and a scale of 1 to 6 points for wrinkles' depth (Figure 3).

\section{Subject self-assessment}

The subject answered the self-evaluation questionnaire as follows:

- at the T0 visit before starting the study Twin Serum treatment regarding their actual skin conditions and also on different items such as dry/flaky skin, greasy (oily) skin, redness, dull complexion, dark patches. 
- at T8 weeks visit for their skin improvement on the same items evaluated at T0 and also their opinion on whether they have firmer skin, visibly reduced lines, less prominent wrinkles, improved skin tone, glowing, more radiant appearance, increased skin smoothness, reduced blemishes and reduced dark circles around eye area.

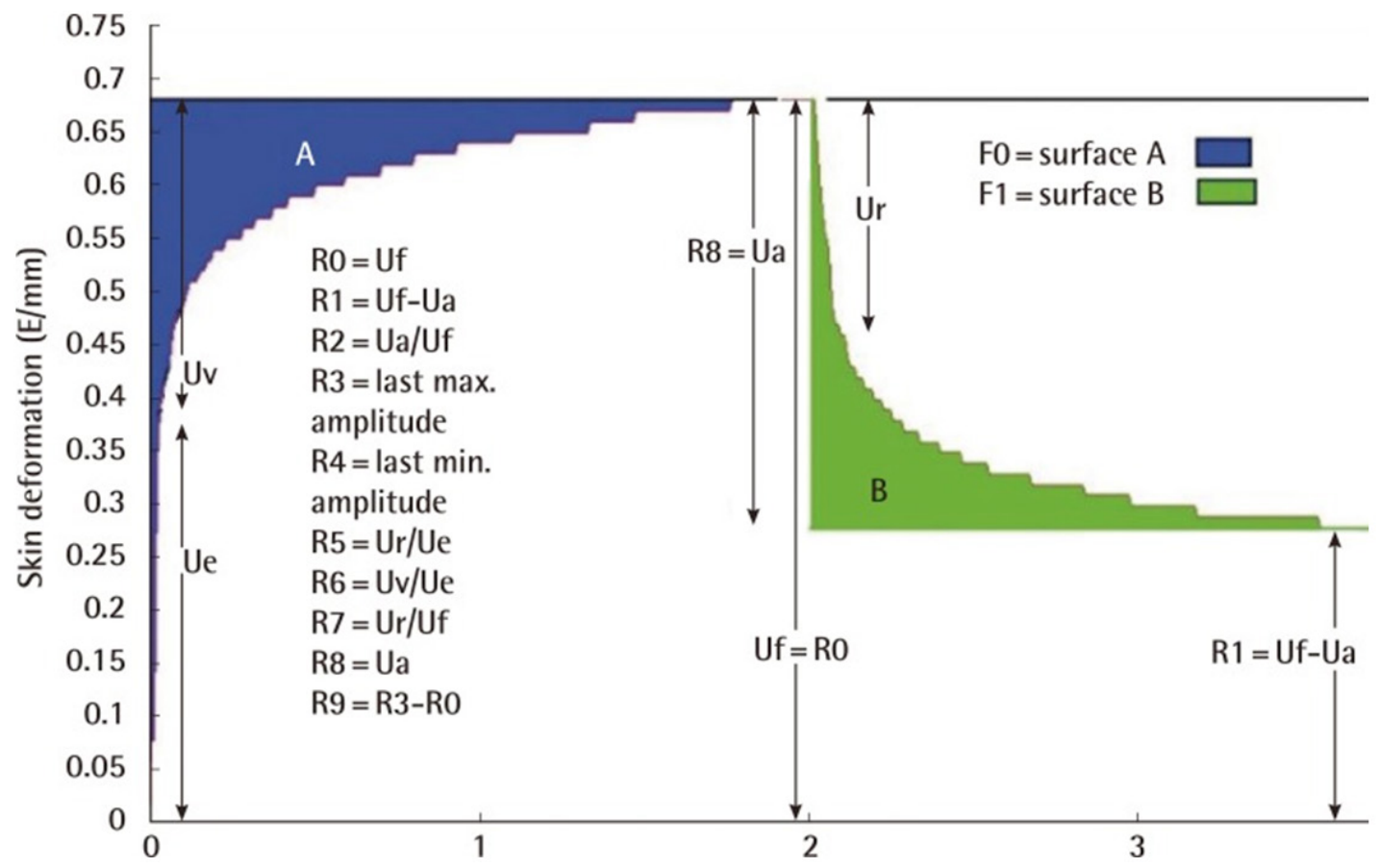

Figure 2 Assessment of skin elasticity and flexibility R0:This parameter looks at the maximum amplitude and represents the passive behavior of the skin to the applied force (firmness). RI/R4:The ability of the skin to return to its original state (minimum amplitude after relaxation). R2: Gross elasticity: resistance versus ability of returning. R3/R9:Tiring effect (fatigue) visible for repeated suction/relaxation. R4: Residual skin deformation after release of the last suction. R5: Net elasticity: elastic portion of the suction part versus the elastic portion of the relaxation part. R6: Portion of the visco-elasticity of the curve. R7: Portion of the elasticity compared to the complete curve. R8: Complete relaxation after the pressure is cut off. Ua, final retraction; Ue, immediate distension; Uf, total deformation; Ur, immediate retraction; Uv, delayed distension.

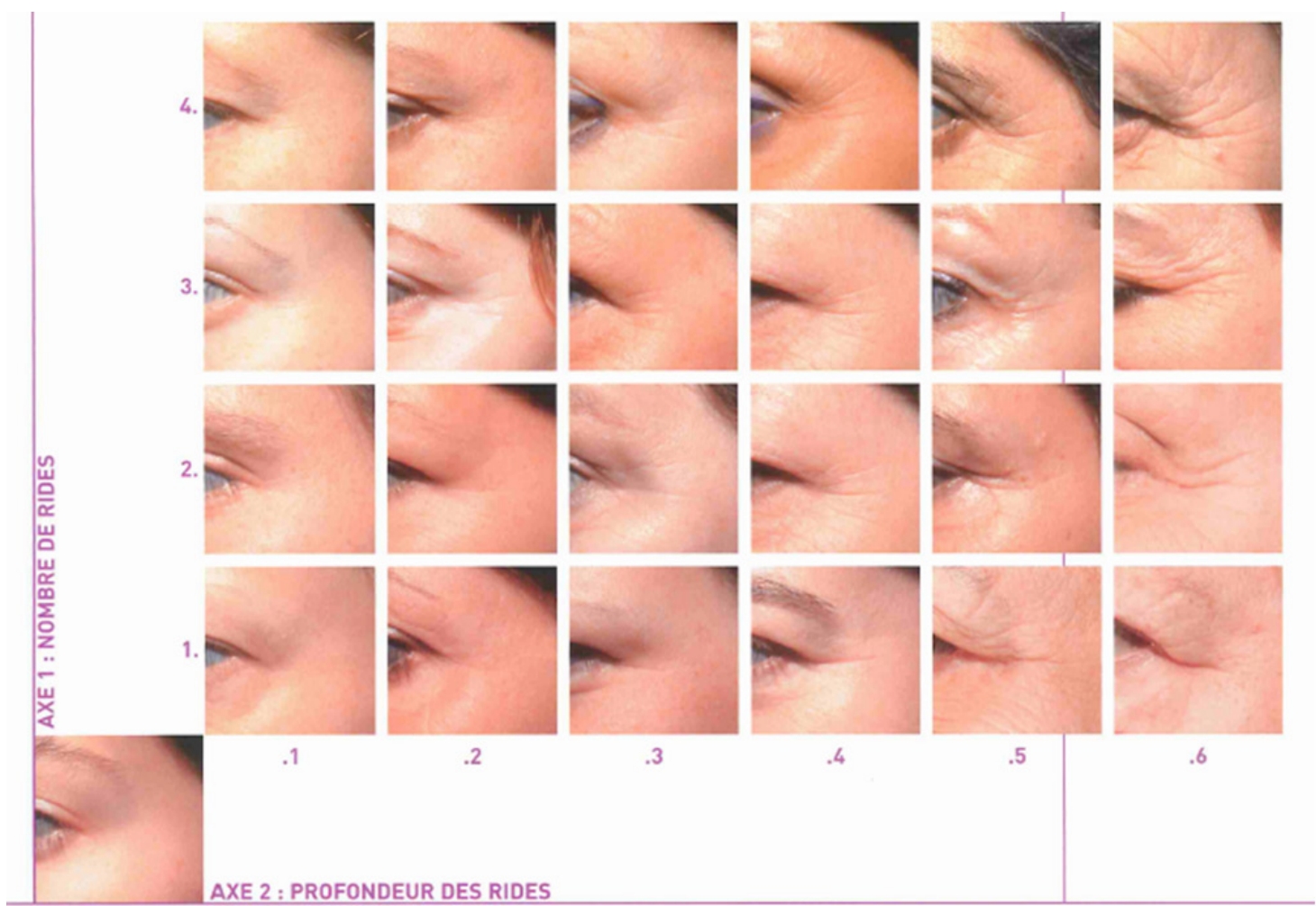

Figure 3 Bazin periorbital wrinkles scale. The vertical axis evaluate the number and the horizontal axis evaluate the depth of the wrinkles.

Citation: Jeudy A, Fanian F, Lihoreau T, et al. Clinical effects of a topical twin-serum (aqua phase and lipo phase) on skin radiance and wrinkle depth: role of active ingredients and delivery optimisation.J Dermat Cosmetol. 202 I;5(3):48-55. DOI: I0. I5406/jdc.202I.05.00 I83 


\section{Statistical analysis}

The statistical analysis was conducted using Statistica ${ }^{\circledR}$ version 7.1 (Statsoft, Tulsa, OK, USA) and Instat GraphPad ${ }^{\circledR}$ version 3.06 (graphpad Software Inc, San Diego, CA, USA) software.

The analyses were based on the intention-to-treat (ITT) population. Descriptive statistics were reported for each parameter, i.e., mean, standard deviation, minimum, maximum, and median, quartiles for quantitative data, and percentages for qualitative data.

Quantitative variables were tested by a Student test or Wilcoxon test depending on the normality of the distribution, tested by a Shapiro-Wilk test. Statistical significance was set at $\mathrm{P}$-value $\leq 0.05$ and at $\mathrm{P}$-value $0.05<\mathrm{p}<0.10$ for trends

\section{Results}

30 healthy volunteers aged 36-61 years (mean $49.5 \pm 6.7$ years) were enrolled. All subjects completed the study. The compliance was reported to 0.7 missed applications on average, per volunteer; $(\max =7 ; \min =1)$.

\section{Skin radiance}

For the color parameters of C.L.B.T. ${ }^{\mathrm{TM}}$ scoring, statistical analyses revealed a significant decrease of Pink Red (38\%), Olive (31\%), Beige $(30 \%)$, Light Pink (21\%) parameters $(\mathrm{p}<0.001)$. Analyses have shown that the Luminosity, Transparency, Brightness increased significantly $(\mathrm{p}=0.001)$ after 8 weeks of application by $29 \%, 28 \%$ and $27 \%$ respectively (Figure 4 ).

\section{Pink Red}

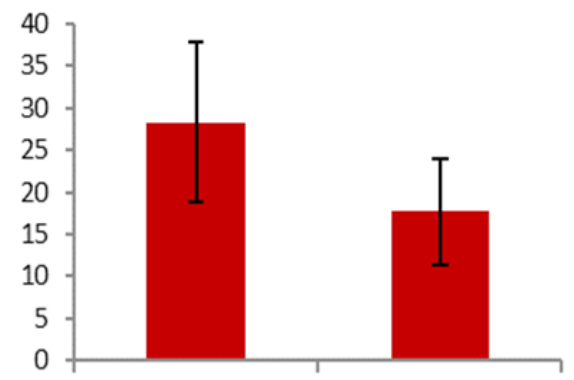

TO

$\mathrm{T} 1$
Luminosity

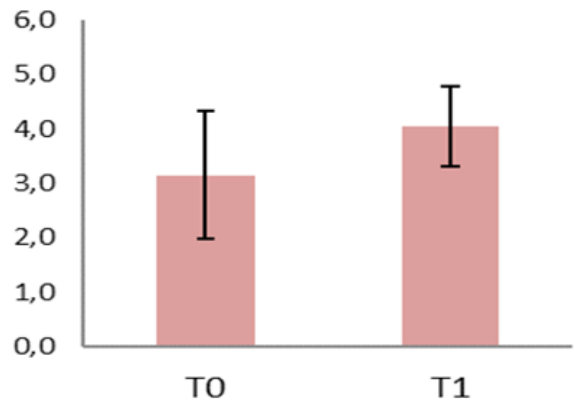

Olive

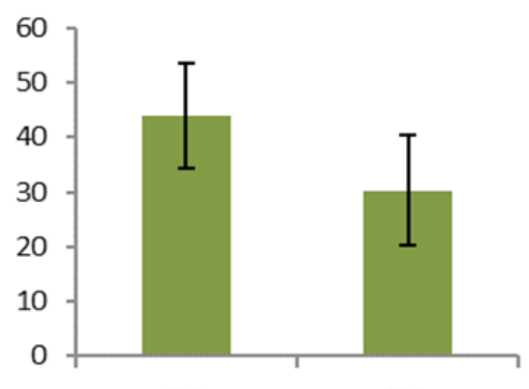

TO
Beige

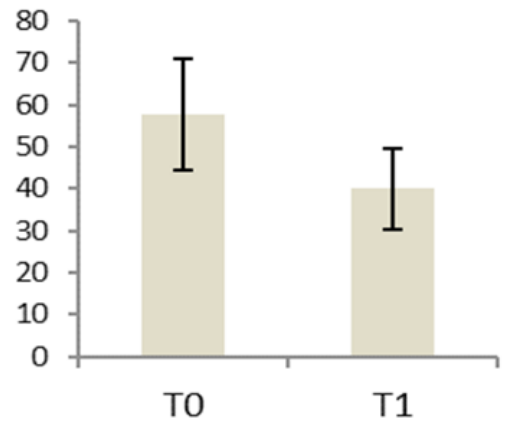

Transparency

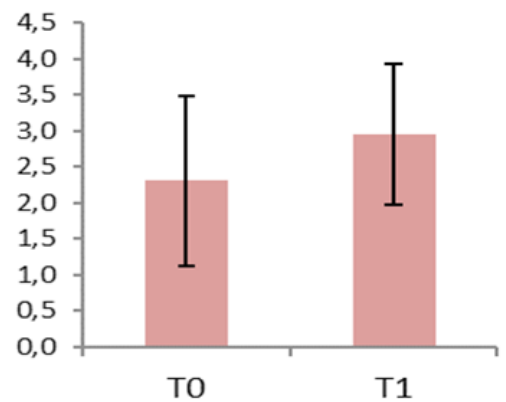

Figure 4 Evaluation of C.L.B.T.TM parameters at T0 and TI. *** $<<0.00$ I. Pink Red, Olive and Beige were significantly decreased at TI (to T0). Luminosity, Brightness and Transparency were significantly increased at TI (to T0).

No significant evolution was observed on the Glossymeter ${ }^{\circledR}$ parameters, after 8 weeks.

\section{Skin elasticity}

An increase which tends to be significant $(\mathrm{p}=0.06)$ was observed for the $\mathrm{Ur} / \mathrm{Ue}$ parameter from $\mathrm{T} 0(0.462 \pm 0.098)$ to $\mathrm{T} 8$ weeks $(0.506 \pm 0.111)$ which indicates an important improvement of skin elasticity by $9.5 \%$. The firmness and fatigability parameters did not show any statistically significant evolution between T0 and T8weeks.

\section{Crow's feet wrinkles}

Crow's feet wrinkles were evaluated by Bazin visual clinical scoring (Figure 3 ) in 2 axis : 1 to 4 scale for wrinkled number ( 1 for no wrinkle and 4 for too many wrinkles) and 1 to 6 scale for wrinkles depth ( 1 for no wrinkle and 6 for very deep wrinkles). The results revealed that the number of wrinkles from grade 3 (9 versus 4 ) was evolved to grade 2 ( 8 versus 12 ). In addition, the wrinkles depth was evoluted from grades 6 and 5 (20 versus 13) to grade 4 (5 versus 12) (Figure 5).

\section{Subject self-assessment}

After 8 weeks of topical application of a two phases Twin Serum, the subjects found their skin less dull $(\mathrm{p}=0.002)$, less red $(\mathrm{p}=0.002)$, with less spots $(\mathrm{p}=0.044)$, less sagging $(\mathrm{p}=0.002)$ and less wrinkles $(\mathrm{p}=0.002)$ (Figure 6).

In addition, $90 \%$ of subjects experienced at least "some improvements" to "significant improvement" on their skin and $60 \%$ of subjects had comments on the improvements noticed by their friends. $90 \%$ were "satisfied" with the study product compared to other products previously used. $73 \%$ of subjects would like to continue using the study product (Perfectil ${ }^{\circledR}$ Twin Serum). 
Wrinkles - number

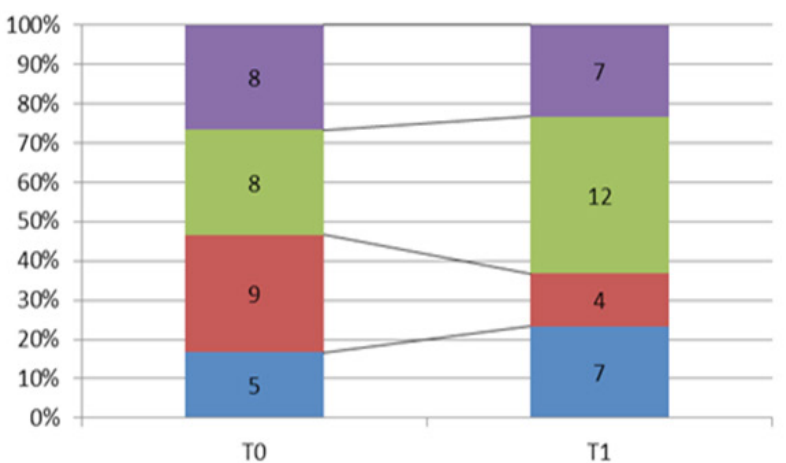

Wrinkles - depth

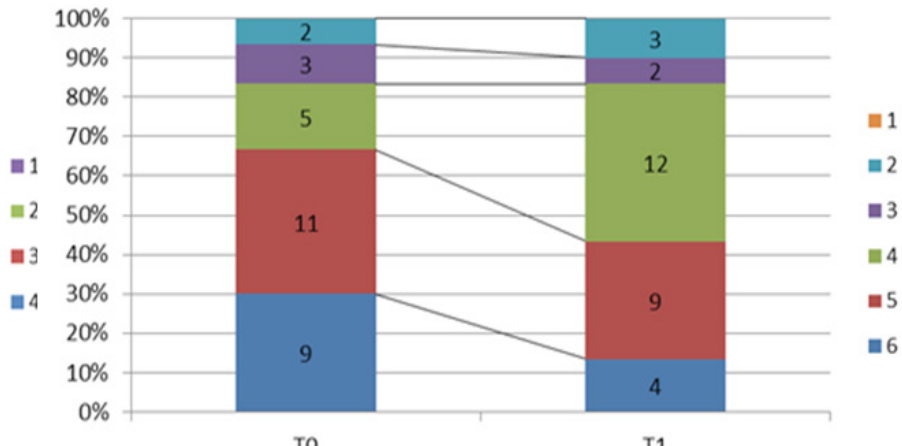

T0

Figure 5 Evaluation of wrinkles by clinical scoring at T0 and TI ( 8 weeks). The depth of wrinkles was significantly decreased at TI versus T0 $(* *$ p $=0.003)$.

Dull complexion

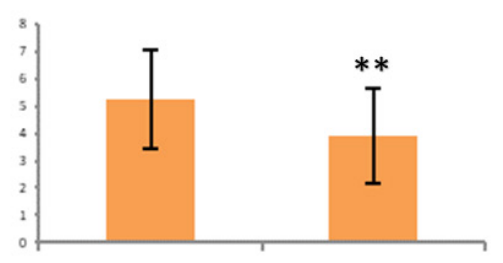

TO

T1
Sagging skin

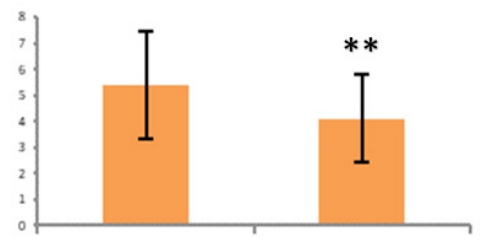

TO

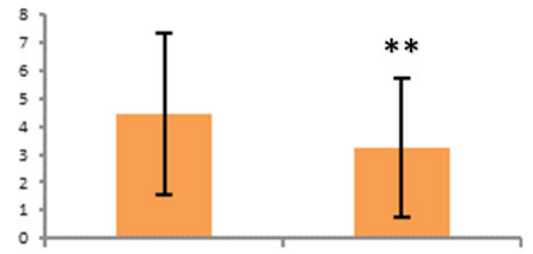

TO
Redness

T1
Deep wrinkles

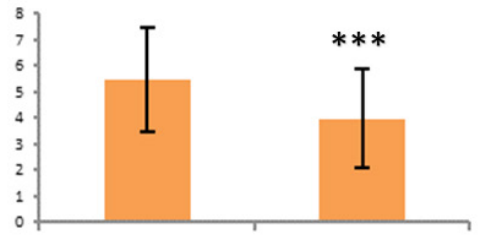

T0

T1

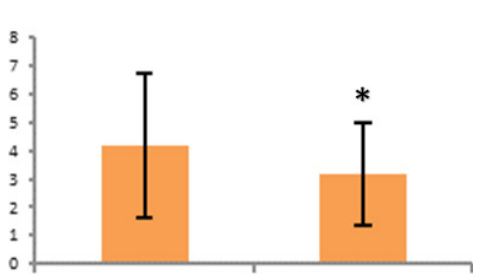

$\mathrm{T} 1$

Figure 6 Auto evaluation at T0 andTI. ${ }^{*} \mathrm{p}<0.05 ;{ }^{*} \mathrm{p}<0.01 ;{ }^{* * *} \mathrm{p}=0.00 \mathrm{I}$. Dull complexion, redness, spot, sagging skin, deep wrinkles, fine wrinkles were significantly decreased at TI (to T0).

\section{Safety analysis}

No serious adverse event has been reported. The tolerance of the product was good. Only 6mild intolerance cases were reported: erythema for 2 subjects, tingling, peeling, pimples and bloating for 1 subject. All these intolerances were resolved without intervention and did not cause the discontinuation of the study.

\section{Discussion}

This study took place at the CERT (Center for Study and Research on the Integument) situated at the Department of Dermatology, University Hospital of Besançon, France between September, and November. This centre is certified by the French Ministry of Research.

30 female volunteers with moderate to severe facial skin photoaging (grade 3 to 4 based on Glogau photoaging scale) participated in this open label, non-comparative study, to investigate the effects of a topical double phaseserum, Perfectil ${ }^{\mathbb{B}}$ Twin Serum, VITABIOTICS Ltd, London, UK.

The products were applied twice daily during 2 months. The volunteers were examined at T0 (before treatment) and at T8 weeks.
All measurements took place in a temperature- and humidity-regulated environment.

The product contains a bi-phasic serum (one Lipo phase named Lipo Serum and the other Aqua phase named Aqua Serum) presented in 2 separated bottles which should be mixed in the palm of the hands immediately before application. This mixing procedure lets the product become a homogenous emulsion at body temperature which can be absorbed visibly and immediately to the skin.

The results revealed a significant improvement in all indicators of skin radiance as the main objective, after 2 months. Indeed, the clinical scoring C.L.B.T. ${ }^{\mathrm{TM}}$ has shown an improvement in skin radiance through an increase of Luminosity, Transparency, Brightness while a decrease of Pink Red, Olive, Beige, Light Pink parameters. Dumoulin et al. ${ }^{21}$ used the same method to evaluate the effects of an oral supplementation on skin radiance. The comparison of the results shows that although the oral supplementation rich in antioxidants could significantly modify the skin radiance complexion but a topical treatment is more effective particularly on the skin transparency and brightness (Table 1). 
Table I comparative data: effects of the topical bi-phasic serum compared to an oral supplementation on skin radiance parameter

\begin{tabular}{lll}
\hline & Current study & Dumoulin et $\mathbf{~ a l}^{2 \mathbf{1}}$ \\
\hline Luminosity & $+29 \% * *$ & $+25.9 \% * * *$ \\
Transparency & $+28 \% * *$ & $\mathrm{NS}$ \\
Brightness & $+27 \% * *$ & $-12.3 \%$ \\
Red pink & $-38 \% * *$ & $-34.8 \% * * *$ \\
Olive & $-31 \% * *$ & $-21.6 \% * * *$ \\
Beige & $-30 \% * *$ & NS \\
Light pink & $-21 \% * *$ & Not available \\
\hline
\end{tabular}

$*_{p}<0.05 ; * * p<0.01 ; * * * p<0.001$

This result could be argued by the direct effects of the topical product on the surface of the skin which directly reflects the light. Another argument could be the combination of several ingredients which have been proved to modify the skin color/radiance. These ingredients are as follow: Lactic Acid in "aqua phase" and Retinyl Palmitate, Vit E and C in "Lipo phase". In addition, the presence of Lecithin as an emulsifier with a good Hydrophile-Lipophile Balance could provide a suitable galenic form. Niacinamide (NIA), also known as nicotinamide, is a hydrophilic amide of vitamin B3 that is an important component in various cosmetics and medicines. ${ }^{22}$ NIA blocks the melanosome migration between melanocytes and keratinocytes and suppresses skin pigmentation. ${ }^{22-23}$ The biological activities of NIA also include antimicrobial, photo-protection, lighting, anti-inflammation and anti-pruritus. ${ }^{25}$ Therefore, it could be considered as an effective ingredient for skin radiance.

A statistically significant decrease was observed over time for the wrinkles' depth (through clinical scoring), which shows an improvement in skin relief. These results are explicable with the presence of Retinyl palmitate and also Vit E and $\mathrm{C}$ as the well-known anti-oxidants.

For the elasticity, an improvement which tends to be significant ( $\mathrm{p}=0.06$ ) for the Ur/Ue parameter, was observed which was coherent with the subjects' self-assessment results. The sagging skin was diminished significantly $(\mathrm{p}=0.002)$ after 8 weeks of application of the Twin serum. The elasticity evolution is normally a long term effect and needs at least 3 to 6 months. However the results of this study revealed that the biphasic nature of the product could help the skin to remodel itself over time.

Although previous studies have shown that winter seasonal changes could have negative effects on the structural components of skin, the results of this study showed that this product could help the skin in order to face these negative effects. Fanian et al (2013) showed that the free fatty acids, linoleic acid, some saturated fatty acids, oleic acid, stratum corneum lipids, ceramide 1 linoleate, and ceramide 1 oleate, as well as biometrologic parameters, including skin water content, transepidermal water loss, and skin sebum could be influenced by the seasonal changes. ${ }^{26}$ This data could confirm that restoring these ingredients to the skin could protect it against these negative changes.

The subjects' self-assessment results revealed a significant improvement in skin radiance, skin redness, spot, sagging skin, and the wrinkles (deep and fine). In addition, $90 \%$ of subjects experienced at least "some improvement" and were at least "satisfied" with the Twin serum compared to other products already in use. $73 \%$ of subjects would like to continue using Perfectil Twin Serum, and $60 \%$ of subjects had positive comments by their friends. This data is supportive of the restorative effect of this bi-phasic serum on skin surface structure. In addition, the activation method of the two phases in the palm of the hands to form a liposomal emulsion, without the use of petroleum based oils, before applying the product could help the product to penetrate more deeply in the skin.

\section{Conclusion}

In conclusion, this study could support the effect of a topical biphasic product on the skin's radiance and wrinkle depth after 8 weeks of treatment. These results could be justified by its new approach to mix both phases by warming it and forming a lipid emulsion in the palm of the subject's hand. This action may have a positive effect on the immediate penetration of the product which should be evaluated through the further studies. In addition, the bi-phasic nature of the product could facilitate the targeting of each phase to a dedicated layer, particularly to restore the lipidic layer which give it a specific focused approach.

\section{Conflicts of interest}

The authors declare no conflict of interest.

\section{Acknowledgments \& funding}

Authors keep the memory of Dr Ahmed Elkhyat who has participated very actively in all steps of this scientific project from concept to the manuscript final revision but unfortunately, he passed away before publication of this article.

This research paper is made possible through the help and support from everyone, including: patients, technicians and research engineers of our center, especially Mrs Celine THIEBAUT, Mrs Aurelie DURAI and Mrs Vanessa ECARNOT for their appreciable enthusiasm to carry out the exact measures. The study was commissioned by Vitabiotics Ltd. company.

\section{References}

1. Fink B, Grammer K, Thornhill R. Human (Homo sapiens) facial attractiveness in relation to skin texture and color. J Comp Psychol Wash DC. 2001;115(1):92-99.

2. Fink B, Matts PJ. The effects of skin colour distribution and topography cues on the perception of female facial age and health. $J$ Eur Acad Dermatol Venereol JEADV. Avr. 2008;22(4):493-498.

3. Samson N, Fink B, Matts PJ. Visible skin condition and perception of human facial appearance. Int J Cosmet Sci. 2010;32(3):167-184.

4. Samson N, Fink B, Matts PJ, et al. Visible changes of female facial skin surface topography in relation to age and attractiveness perception. $J$ Cosmet Dermatol. 2010;9(2):79-88.

5. Samson N, Fink B, Matts P. Interaction of skin color distribution and skin surface topography cues in the perception of female facial age and health. J Cosmet Dermatol. 2011;10(1):78-84.

6. Nkengne A, Bertin C, Stamatas GN, et al. Influence of facial skin attributes on the perceived age of Caucasian women. J Eur Acad Dermatol Venereol JEADV. 2008;22(8):982-991.

7. Jones AL, Porcheron A, Sweda JR, et al. Coloration in different areas of facial skin is a cue to health: The role of cheek redness and periorbital luminance in health perception. Body Image. 2016;17:57-66. 
8. Fink B, Matts PJ, Klingenberg H, et al. Visual attention to variation in female facial skin color distribution. J Cosmet Dermatol. 2008;7(2):155-161.

9. Matsubara A, Liang Z, Sato Y, Uchikawa K. Analysis of human perception of facial skin radiance by means of image histogram parameters of surface and subsurface reflections from the skin. Skin Res Technol. 2012;18(3):265-271.

10. Petitjean A, Sainthillier J-M, Mac-Mary S, et al. Skin radiance: how to quantify? Validation of an optical method. Skin Res Technol. 2007;13(1):2-8

11. Purdue GF, Hunt JL. Cold injury: a collective review. J Burn Care Rehabil. 1986;7(4):331-42.

12. Middleton JD. The mechanism of water binding in stratum corneum. $\mathrm{BrJ}$ Dermatol. 1968;80(7):437-450.

13. Monfrecola G, Riccio G, Savarese $\mathrm{C}$ et al. The acute effect of smoking on cutaneous microcirculation blood flow in habitual smokers and nonsmokers. Dermatol Basel Switz. 1998;197(2):115-118.

14. Koh JS, Kang H, Choi SW, Kim HO. Cigarette smoking associated with premature facial wrinkling: image analysis of facial skin replicas. Int $J$ Dermatol. 2002;41(1):21-27.

15. Bensé I, Clot J, Misery L, et al. Stress et dermatologie. In: Stress, pathologies et immunité. Médecine-Sciences Flammarion. Paris: Thurin JM, Baumann N. 2003; p.192-199.

16. Petitjean A. Approches biométrologiques de l'éclat du teint. Sci Vie Santé 2006.

17. Jeudy A, Mac-Mary S, Sainthillier J-M, et al. Skin Radiance Measurement In: Handbook of Cosmetic Science and Technology. Fourth Edition. New York : Edition Informa healthcare: Barel AO, Paye M, Maibach HI; 2014 p. 459-66.
18. Baret M, Bensimon N, Coronel S, et al. Characterization and quantification of the skin radiance through new digital image analysis. Skin Res Technol. 2006;12(4):254-260.

19. Jeudy A, Ecarnot V, Humbert P. Measurement of skin radiance. In: Agache's Measuring the Skin. 2nd edn, Springer. Humbert P, FANIAN F Maibach HI. 2017. p.161-76.

20. Musnier C, Piquemal P, Beau P, et al. Visual evaluation in vivo of « complexion radiance » using the C.L.B.T. sensory methodology. Skin Res Technol. 2004;10(1):50-56.

21. Dumoulin M, Gaudout D, Lemaire B. Clinical effects of an ora supplement rich in antioxidants on skin radiance in women. Clin Cosmet Investig Dermatol. 2016;9:315-24.

22. Zhen AX, Piao MJ, Kang KA, et al. Niacinamide Protects Skin Cells from Oxidative Stress Induced by Particulate Matter. Biomol Ther. 2019;27(6):562-569.

23. Bissett DL, Robinson LR, Raleigh PS, et al. Reduction in the appearance of facial hyperpigmentation by topical $\mathrm{N}$-acetyl glucosamine. J Cosmet Dermatol. 2007;6(1):20-26.

24. Kimball AB, Kaczvinsky JR, Li J, et al. Reduction in the appearance of facial hyperpigmentation after use of moisturizers with a combination of topical niacinamide and $\mathrm{N}$-acetyl glucosamine: results of a randomized, double-blind, vehicle-controlled trial. $\mathrm{Br} J$ Dermatol. 2010;162(2):435-441

25. Wohlrab J, Kreft D. Niacinamide - mechanisms of action and its topical use in dermatology. Skin Pharmacol Physiol. 2014;27(6):311-315.

26. Fanian F, Mac-Mary S, Jeudy A, et al. Efficacy of micronutrient supplementation on skin aging and seasonal variation: a randomized, placebo-controlled, double-blind study. Clin Interv Aging. 2013;8:15271537. 